\title{
Arthroscopic patellofemoral denervation in the treatment of severe patellofemoral arthritis.
}

\author{
Jun Tao ${ }^{1}$, Pu Chen ${ }^{2}$, Jie Chen ${ }^{3}$, Lifeng Xie ${ }^{1}$, Li Liu ${ }^{1}$, Yongpeng Yang ${ }^{1}$, Hui Ming Guo ${ }^{\text {* }}$ \\ ${ }^{1}$ The Second Affiliated Hospital of Nanchang University, PR China \\ ${ }^{2}$ Feng Cheng People's Hospital, PR China \\ ${ }^{3}$ The Third Clinical Medical College of Nanchang University, PR China
}

\begin{abstract}
Objective: To evaluate the effect of arthroscopic patellofemoral denervation in the treatment of severe patellofemoral arthritis.

Methods: 60 cases with anterior knee pain, accompanied with severe pain when walking up and down the stairs or squatting in in our hospital from January 2012 to July 2015 were recruited for arthroscopic patellar denervation treatment, 24 of them were male and 36 female. Their average age was 58.7, and the average course of disease was $11.3 \mathrm{y}$. After 6 months and 12 months of the treatment, FELLER patellar score were compared with preoperative patellar score, and knee joint $\mathrm{CT}$ was conducted to assess patella tracking correction. Complications such as medial patellar dislocation, reflex dystrophy, ischemic necrosis and knee weakness were observed.

Results: The average follow-up time was $\mathbf{2 6 . 5}$ months. No complications such as infection or nerve and blood vessel injury, medial patellar dislocation and weakness of knee extension were found. Before and after surgery, FELLER patellar score was adopted to measure patellar tilt angle, there was a significant difference between scores before and after surgery, $P \leq 0.05$.

Conclusions: Patellofemoral denervation arthroscopic treatment can help patella formation and release lateral retinaculum to relieve and improve the symptoms and achieve the goal of treatment.
\end{abstract}

Keywords: Arthroscopy, Patellofemoral joint, Denervation, Patellofemoral arthritis.

Accepted on November 17, 2017

\section{Introduction}

Gonitis is the most common form of osteoarthritis, and it has been reported that $67 \%$ of the patients with gonitis manifest simple patellofemoral arthritis. For the patients with patellofemoral arthritis who have not been cured by conservative treatment, TKA (Total Knee Replacement) or PFA (Patellofemoral Joint Replacement) is now adopted. However, for the relatively young patients (prosthesis life and process limitations lead to revision arthroplasty in patients with life expectancy) and patients untolerable trauma, knee replacement is not the best method, and joint replacement needs high cost and big surgical trauma. We investigated whether arthroscopic patellofemoral denervation for the treatment of severe patellofemoral arthritis can be a novel alternative therapy to compensate for above deficiency to reduce surgical costs and trauma and delay joint replacement age.

\section{Subjects and Methods}

\section{General information}

Ethical approval was given by the medical ethics committee of Second affiliated hospital of Nanchang university with the following reference number: 2011014. 60 cases with anterior knee pain, accompanied with severe pain when walking up and down the stairs or squatting in in our hospital from January 2012 to July 2015 were recruited for arthroscopic patellar denervation treatment, 24 of them were male and 36 female. Their average age was 58.7, and the average course of disease was $11.3 \mathrm{y}$. Before surgery, the knee joint CT scanning was conducted, and the inclination of the patella was measured. The saga sign $(+)$ suggested that the patella had an outward tendency to move outwards. Inclusion criteria: 1 . in accordance with the operation indications for treatment, symptoms markedly, life and work affected, no obvious improvement after 3 months of conservative treatment; 2 . age $\geq 50$ ye; 3 . a clear diagnosis based on the clinical manifestation and auxiliary examination, Kellgren Laurence grade $\geq$ II level; 4 . the tibial joint with relatively mild symptoms, no obvious pain 
when walking; 5. the patient's informed consent, and followup. Exclusion criteria: 1. did not meet the inclusion criteria; 2. accompanied with obvious knee ligament injury, meniscus removal, congenital patella anatomic abnormalities associated with vascular nerve injury; 3 . other diseases influencing the results, such as myasthenia gravis; 4. the single leg standing knee radiograph examination showed severe lower limb misalignment, 5. poor compliance.

\section{FELLER patellar score}

The proportion of anterior knee pain in the FELLER patella score was up to $50 \%$. Other indicators included the quadriceps strength, the ability to stand up from the chair, and the ability to move up and down the floor, making a better assessment of the function of the patella (Table 1).

Table 1. FELLER patellar score.

\begin{tabular}{ll}
\hline Items & Score \\
\hline Anterior knee pain & No 15 points, mild 10 points, moderate 5 points, severe 0 point \\
\hline Quadriceps strength & Excellent (5/5) 5 points, good (4/5) 3 points, poor (3/5) 1 point \\
\hline $\begin{array}{l}\text { Standing up from the } \\
\text { chair }\end{array}$ & Be able to (without hands) 5 points, Be able to (with hands) 3 points \\
\cline { 2 - 2 } & Be able to, but with difficulty 1 point, be not able to 0 points \\
\hline $\begin{array}{l}\text { Going up and down } \\
\text { the floor }\end{array}$ & $\begin{array}{l}\text { Going up and down the floor without hands } 5 \text { points, going up and down the floor with hands } 4 \text { points, cannot go up and down the floor without } \\
\text { support } 3 \text { points, cannot go up and down the floor with support } 2 \text { points }\end{array}$ \\
\hline
\end{tabular}

\section{Methods}

Arthroscopy evaluation: At first, the three compartment knee arthroscopy was conducted to assess the articular cartilage surface. The cartilage lesions were graded according to the Outer bridge method: grade 0 , normal; edema or vesicular structure; grade I, Cartilage softening, edema, or vesicular structure; grade II, thinning of the cartilage, moderate fibrosis or fracture; grade III, cartilage stripping off partly; grade IV, cartilage full-thickness injury or exfoliation, subchondral bone exposure.

The tension of patellofemoral ligament and the hyperplasia of the bone around the patella were observed. Flexion and extension of the knee was used to dynamically assess the patellofemoral congruence, and graded according to the Casscells method. Grade I: when the knee is bent for $60^{\circ}$, the patella is located at the center of the femoral intercondylar pulley; Grade II: when the knee is bent between 60 to 90 degrees, the patella is at the center of the pulley; Grade III: when the knee is bent for 90 degrees, the patella is at the center of the pulley.

Intra-articular patellar lateral plasty with extra articular retinaculum release: The superior patellar lateral approach, the patellar medial and lateral approach are usually used. The drill or radio head was put from the lower lateral approach of the patella, drilling to remove the outer margin of the patella and osteophyte, so that it becomes type I or type II patella. Flexion and extension of the knee was used to dynamically assess the patellofemoral congruence and contracture of the patella ligament. The high pressure lateral patellofemoral, damaged lateral patellar cartilage were given the RF head along the lateral margin of the patella is about $2 \mathrm{~cm}$, loosen the lateral patellotibial ligament (Figure 1), lateral patellofemoral support with shallow and deep loosening, edge evaluation, especially should release into the fat pad of fibrous cord, which can also be in the joint of patella lateral forming and joint relinacular release alternately.

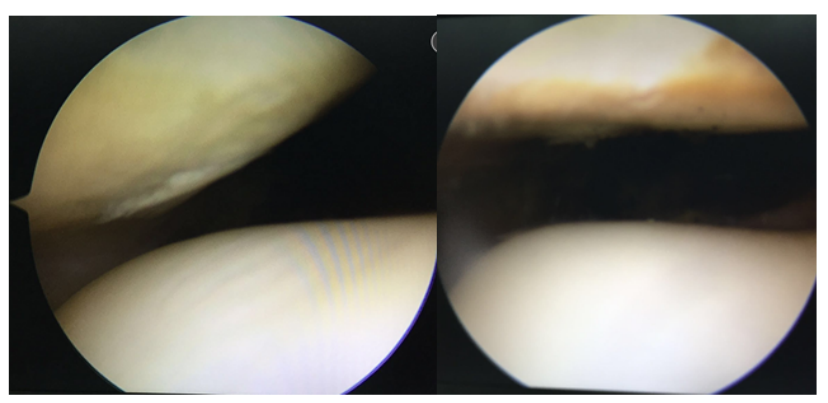

Figure 1. The comparison of patellofemoral joint compartment before and after surgery cartilage formation and radiofrequency denervation of the patella.

The electric shaver or curette was used to treat III and IV degrees of patellar cartilage degeneration, so that the cartilage surface smooth and stable. The old exposed bone surface should be drilled freshly, which is beneficial to the formation of fibrocartilage. The radio-frequency head was along the patellofemoral joint to burn neural processing. The denervation parts and scopes of patellar and femoral condyle should be determined according to the extent of pain and cartilage injury extends (Figure 2); patella nerve fibers were distributed in regional concentration manner (Figure 3). The denervation of the patellar nerve should be clearly done for the medial and inferior tissues.

Treatment of intra-articular complications: After free body removal, meniscus trimming and synovial plica cutting, a joint drainage tube was placed, and the knee was compressed from the outside to the inside to maintain the loosening state of the lateral supporting band. After the operation, the pressure was bandaged for $48 \mathrm{~h}$. Straight leg rising and the pushing patella exercise were performed in the early time. After the drainage tube was removed, the knee flexion exercise was performed. 
Follow-up, functional exercises and surgical results evaluation: At 6 months and 12 months after the operation, the FELLER patella score was compared with that before the operation. The knee joint $\mathrm{CT}$ was taken to evaluate the correction of the patellar trajectory. Complications such as medial patellar dislocation, reflex dystrophy, ischemic necrosis and knee weakness were observed.

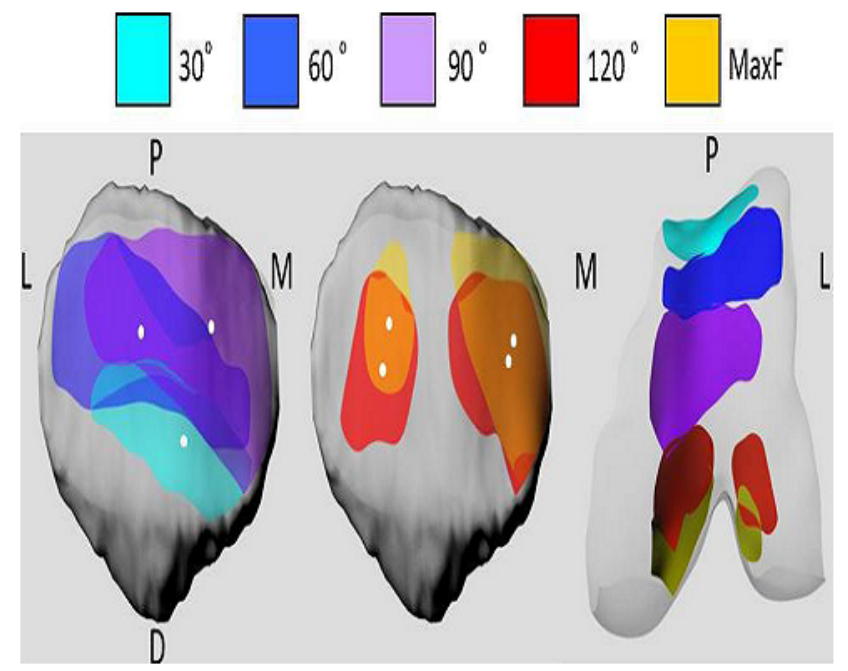

Figure 2. Motion trajectory of the patellofemoral joint.

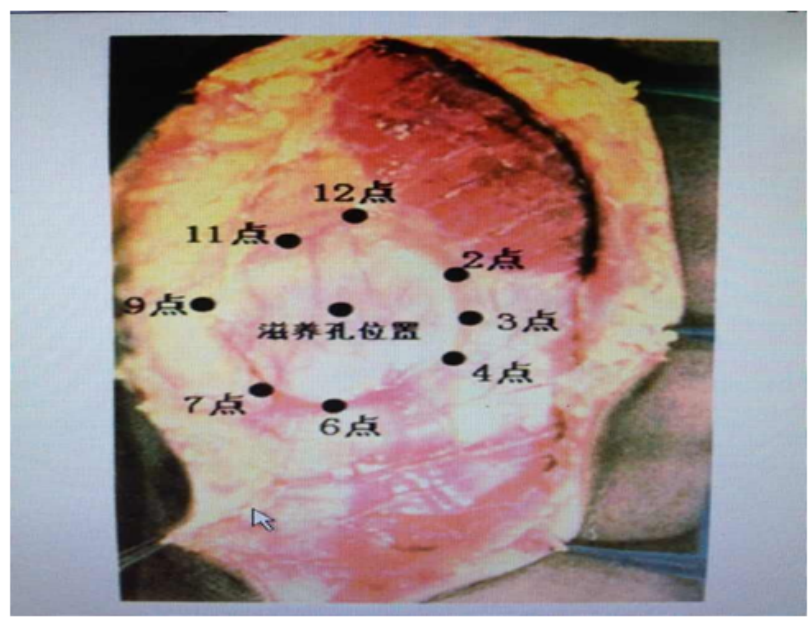

Figure 3. The nerve distribution of the patella.

\section{Statistical analysis}

SPSS13.0 was employed for analysis, measurement data was presented as $\overline{\mathrm{X}} \pm \mathrm{s}$, and $\mathrm{t}$ test was used for comparison. $\mathrm{P} \leq 0.05$ means statistically significant difference.

\section{Results}

All the incisions were healed at I phase, without any complications such as infection, nerve or vascular injury, reflex dystrophy and ischemic necrosis and knee weakness. 59 cases were followed up for 6 57 months with an average of 26.5 months. The knee joint CT at the last follow-up showed no medial patellar dislocation, $0^{\circ}$ position and 30 knee flexion test and the fear test were negative, and all the patient did not touch the cord ligament structure outside the patella. At 3 months after the operation, the knee flexion and extension of the knee joint were all restored to the preoperative level. In 57 cases, the pain was relived than that before operation, and there were 6 cases of patellofemoral friction or snapping. 55 cases considered that the quadriceps strength of the injured side was better than that of the uninjured side, and there was no muscle weakness in the quadriceps strength after operation. In 55 cases, the ability to stand up from the chair was higher than that before the operation, and the ability of up and down stairs was improved in 56 patients. At the last follow-up, the FELLER patella score was $(25.44 \pm 3.25)$ points, and the difference was statistically significant compared with that before operation ( $\mathrm{t}=-4.532, \mathrm{P}=0.031$, Tables 2 and 3 ).

Table 2. Scoring of 59 cases of patellofemoral arthritis pre and post operation.

\begin{tabular}{lll}
\hline & FELLER score & P value \\
\hline Pre-operation & $16.58 \pm 4.47$ & - \\
\hline 6 months post-operation & $26.50 \pm 3.66$ & $\mathrm{P}=0.035$ \\
\hline 12 months post-operation & $25.44 \pm 3.25$ & $\mathrm{P}=0.031$ \\
\hline
\end{tabular}

Table 3. Comparison of patella tilt angle pre and post patellofemoral arthritis operation.

\begin{tabular}{lll}
\hline & Patellar tilt angle & P value \\
\hline Pre-operation & $5.54 \pm 2.86$ & - \\
\hline 6 months post-operation & $8.36 \pm 3.05$ & $\mathrm{P}=0.029$ \\
\hline 12 months post-operation & $8.08 \pm 3.27$ & $\mathrm{P}=0.027$ \\
\hline
\end{tabular}

\section{Discussion}

Anterior knee pain is the most common complaint in the orthopedics department. It is reported by Sarda that in patients with knee osteoarthritis, $67 \%$ of them only manifested simple patellofemoral arthritis. The most common cause of this symptom is Lateral Patellar Crush Syndrome (LPSC) [1]. The tight lateral retinaculum produces high pressure on the patellofemoral lateral surface, leading to cartilage softening and exfoliation, as well as its susceptibility to degeneration and osteoarthritis. Sanchis-Alfonso et al. [2] found that in the lateral patellar retinaculum of patients with patellofemoral arthritis, a large secretion of nerve growth factors results in nociceptive nerve hyperplasia and release of substance P. By $\mathrm{H}$ and E staining, immunofluorescence staining and S100 immunohistochemical staining method, it was observed that the channels of nerve into patella were mainly focused on the synovial layer, fat pad and tendon connective tissue layer near the end of the patella in a regional concentrated manner (Figure 3). Fulkerson et al. [3] carried out biopsy of the lateral retinaculum in patients with patellar pain who had undergone lateral loosening, showing histologic changes comparable to those of Morton neuroma. According to the above reasons, the 
young patients with articular cartilage lesion, high pressure lateral patellofemoral, damaged lateral patellar cartilage damage, loosen the lateral patellotibial ligament, lateral patellofemoral support with shallow and deep, and lift the patella lateral patellar compression molding [4-8], eliminates the mechanical and chemical factors causing symptoms. We propose that the focal areas of the burn are the upper and lower patellar poles and the medial margin of the patella. The lower patellar pole should be burned to the patellar tendon layer and part of the fat pad removed.

However, the mechanism of pain in patients with severe patellar arthritis has long been the focus of controversy and there is no conclusion so far. It has been thought that cartilage damage is the pathological mechanism that causes pain, and now studies have shown that simple cartilage damage itself does not cause pain [9], and that is only possible when cartilage damage reaches subchondral bone. Therefore arthroscopic reconstruction of the lateral patellar joint and release of the lateral patellar retinaculum has a clear effect in relived articular cartilage injury; the value of surgery is controversial in Outbridge III-IV patellar hypertension of articular cartilage.

Because the operation cannot repair the cartilage, the pathological basis of osteoarthritis always exist, the lateral patellar formation combined with the lateral patellar retinaculum release had no definite curative effect $[10,11]$. Some scholars have been able to alleviate the symptoms by arthroscopic radiofrequency ablation of the patellofemoral joint to block the pain pathway [13]. We believe that the range of patellofemoral pain and extent of patellar and femoral cartilage injuries were related to patellar tracking and contact damage of articular cartilage in flexion angle changes of knee joint (Figure 2). During the denervation processing of patellofemoral joint, we should focus on handling the responsibility of lesion area, peri patella and femoral condyle to nerve location and scope based on the pain angle and cartilage injury circumstances, synovial tissue also can remove the synovial tissue around the lesion area.

The treatment of patellofemoral arthritis with denervation of the patellofemoral joint combined with patellar reduction and lateral retinaculum release can relieve the symptoms of the patients, reduce surgical costs and trauma, delay joint replacement age and achieve the treatment purpose. As the number of cases and the time of follow-up are limited, we will further observe and evaluate the effect of the operation.

\section{References}

1. Mrchant A. The lateral patellar compression syndrome. New York: Mc Graw-Hill 1993; 166.

2. Sanchis-Alfonso V, Rosello-Sastre E, Monteagudo-Castro C. Quantitative analysis of nerve changes in the lateral retinaculum in patients with isolated symptomatic patellofemoral malalignment. A preliminary study. Am J Sport Med 1998; 26: 703-709.

3. Wang YB, Wang HF. Under arthroscope, the lateral patellar was cleared to support the treatment of knee osteoarthritis. Chin Orthop Surg J 2003; 12: 829-830.

4. Swird P, Fridén T, Boegard T, Kostogiannis I, Neuman P, Roos H. Association between varus alignment and posttraumatic osteoarthritis after anterior cruciate ligament injury. Knee Surg Sports Traumatol Arthrosc ESSKA 2013; 9.

5. Fulkerson JP, Schutzer SF, Pombby GR. Computerized tomography of the patellofemoral joint before and after lateral release or realignment. Arthrescopy 1987; 3: 19.

6. Panni AA, Qarone M, Patricola A. Long term results of lateral retinacular release. J Arthresc Relat Surg 2005; 27: 526-531.

7. Karargyris O, Mandalia V. Arthroscopic treatment of patellar tendinopathy: use of $70^{\circ}$ arthroscope and superolateral portal. Arthrosc Tech 2016; 5: 1083.

8. Sun HQ, Shen AD, Yang HG. The lateral of the patella of the patella in the arthroscope supports the treatment of the lateral high pressure syndrome of the patella. J Clin Orthop 2010; 13: 681-683.

9. Zhang JB, Chen BC, Wang ZQ. The clinical efficacy evaluation of the treatment of patellar femoral arthrosis was supported by the lateral support of arthroscope. China J Endosc 2008; 14: 1152-1155.

10. McKeever DC. Patellar prosthesis. Bone Joint Surg (Am) 1955; 37: 1074-1084.

11. Gupta S, Augustine A, Horey L. Electrocautery of the patellar rim in primary total replacement: beneficial or unnecessary. Bone Joint Surg Br 2010; 92: 1259-1261.

12. Liu XL, Li BL, Wang H, Wu RT, Li JK, Peng ZR. The curative effect of the treatment of severe knee osteoarthrosis was treated with arthroscopy. Chinese Contemp Med 2016; 23.

13. Vermesan D, Prejbeanu R, Laitin S, Damian G, Deleanu B, Abbinante A, Flace P, Cagiano R. Arthroscopic debridement compared to intra-articular steroids in treating degenerative medial meniscal tears. Eur Rev Med Pharmacol Sci 2013; 23.

\section{*Correspondence to}

Hui Ming Guo

The Second Affiliated Hospital of Nanchang University

PR China 